\title{
Sexual Function and Continence in A Urinary Diversion-Undiversion Case
}

\author{
Garofalo $\mathrm{M}^{1}$, Conti GM*1, Mineo Bianchi $\mathrm{F}^{1}$, Gentile $\mathrm{G}^{2}$, Bianchi $\mathrm{L}^{1}$, Saraceni $\mathrm{G}^{3}$, Colella $\mathrm{A}^{1}$, Colombo $\mathrm{F}^{2}$ and \\ Brunocilla $\mathbf{E}^{1}$
}

${ }^{1}$ Department of Urology, Sant'Orsola Hospital - University of Bologna, Italy

${ }^{2}$ Andrology-Unit, Sant'Orsola Hospital - University of Bologna, Department of Urology, Italy

${ }^{3}$ Complex Pelvic Surgery Unit, Sant'Orsola-Malpighi Hospital, Italy

Received: 眥: November 19, 2018; Published: 制: November 27, 2018

*Corresponding author: Conti GM, Department of Urology, Sant'Orsola Hospital - University of Bologna, Bologna, Italy

\begin{abstract}
The case report present the case of a young men with bladder cancer that previously underwent radical cistectomy with orthotopic neobladder $(\mathrm{ON})$, converted to ileal conduct due to early surgical complications, who demanded a further ON conversion because of a perceived strong altered self-body image to affect QoL hugely.
\end{abstract}

Keywords: Radical Cystectomy; Health Related Quality of Life; Urinary diversion; Ileal Conduit; Neobladder

Abbreviations: RC: Radical Cystectomy; BC: Bladder Cancer; UD: Urinary Diversions; ON: Orthotopic Neobladder; IC: Ileal Conduit; HR-QoL: Health Related- Quality of Life; CT: Computed Tomography; EF: Erectile Function

\section{Introduction}

Radical cystectomy (RC) currently represents the gold standard of treatment for non-metastatic muscle invasive bladder cancer (BC) [1]. Over the last decades the complexity of urinary derivations has changed dramatically, from a simple technique aimed to divert urine to complex surgical intervention allowing a normal voiding pattern through the native urethra [2]. BC patients undergoing RC should be offered with different types of urinary diversions (UD), according to oncologic prognosis of disease, technical feasibility, patients' age and comorbidities. Younger patients with a non-trigonal or urethral urothelial cancer, a good life expectancy, with a preserved renal and liver function should be offered with orthotopic neobladder (ON) [3]. ON ileal reconfiguration is a highly challenging and time-consuming procedure, with a high rate of early and long-term complications [4,5]. On the other hand, Ileal Conduit (IC) represents the most worldwide diffusion type of UD, with shorter operative time, fewer post-operative complications and lower incidence of metabolic disorders $[2,6]$.

Regardless the type of UD, RC represent a sort of "mutilation" for the patient and it could affect psychological behavior with strong impact in quality of life, especially in case of incontinent diversion [3]. Therefore, the Health Related- Quality of Life (HR-QoL) is an important concept, recently developed to define patients' health taking into account emotional, psychological, physical aspects and their social interactions. Multiple validated questionnaires have been used to assess HR-QoL in patients who underwent RC, with different items according to the type of UD [7]. We aim to present the case of a young BC men that previously underwent RC with $\mathrm{ON}$, converted to IC due to early surgical complications, who demanded a further ON conversion because of a perceived altered self-body image and poor QoL.

\section{Case Presentation}

A 46-year-old man with IC diversion referred to our department in 2008 with the diagnosis of a $3 \mathrm{~cm}$ lower pole right renal stone, with recurrent back pain and hydronephrosis. The patient had no co-morbidity besides essential hypertension and obesity (BMI 34). Concerning urological history, in 1998, at the age of 35 years old, the patient underwent RC (pathologic examination: transitional cell carcinoma, pT4 N0 R0 Mx and Grade 3) with ON reconstruction at a different tertiary care urologic institute: a $55-\mathrm{cm}$ distal ileal segment was isolated and reconfigured into a " $U$ " fold to reproduce the standard Studer technique [8]. On the 12th postoperative day the patient was submitted to a further surgical procedure to convert the ON into IC urinary diversion due to an entero-urinary fistula. Intraoperative exploration of the abdomen revealed the presence 
of two surgical dehiscences of the reservoir with no evidence of any damage at the level of neo-bladder neck. The surgeon detached the ON from the urethral stump and tried to connect the reservoir to the abdominal wall to perform an IC.

Due to the fixity and the insufficient length of the $\mathrm{ON}$, the surgeon needed to isolate and add a further $7 \mathrm{~cm}$ ileal tract to the ON, to perform the IC. The next hospital stay proceeded with no further complications. The patient subsequently underwent 2 cycles of adjuvant chemotherapy. In 2008, the patient performed a computed tomography (CT), which detected a $25 \mathrm{~mm}$ stone at the lower pole of the right kidney and no evidence of disease's recurrence was identified. The patient referred to our center for the renal stone's treatment and in May he underwent percutaneous nephrolithotripsy for treatment of renal stone increased to $3 \mathrm{~cm}$, with complete resolution. During following diagnostic evaluations, the patient reported a fully preserved sexual potency without assumption of PDE-5 inhibitor or intracavernous injection of prostaglandin but he reported a low number of sexual intercourses, due to his altered body image. The patient expressed a deep dissatisfaction about the impaired quality of life related to the presence of a cutaneous stoma. Considering his young age, strong motivation, a preserved renal function, good performance status and no evidence of recurrence of disease, the patient was proposed a further IC into an ON surgical conversion.

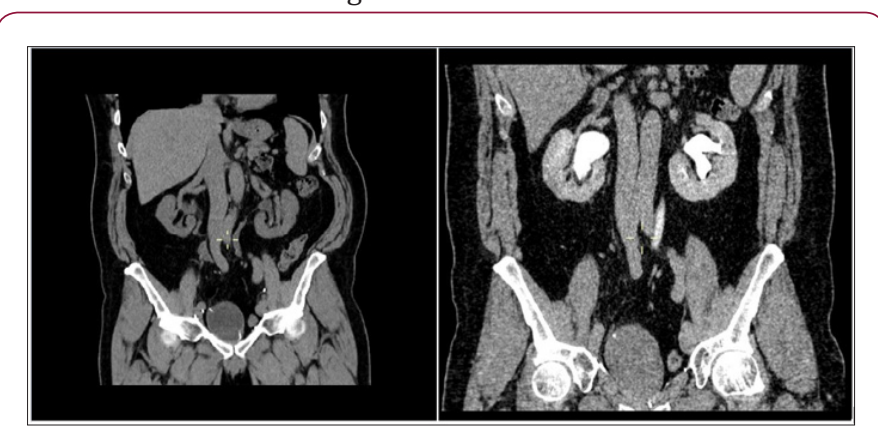

Figure 1:

Note:
a) $\mathrm{CT}$ scan with evidence of novel orthotopic neobladderand
b) regular renal excretory phase with bilateral grade 1 hydronephrosis.

Pre-operative urethroscopy revealed the persistence of urethral lumen up to the urinary sphincter, which appeared still preserved and contractile (Figure 1). The abdominal contrast enhanced CT revealed no ureteral obstruction, with the evidence of a $40 \mathrm{~cm}$ ileal reservoir and no significant hydronephrosis. In May 2009, the patient underwent a further surgical intervention consist of mobilization of the ileal conduit and the reconfiguration of the residual ileal tract in a novel J pouch, leaving intact both the uretero-ileal anastomosis. Then, the distal tract of the J pouch was connected to the urethral stump using non-absorbable suture with separated stitches. No intra- or post-operative complications were recorded. The naso-gastric tube was removed on postoperative day 3 and defecation was signaled on postoperative day 7. The patient was discharged on postoperative day 10. Bladder catheter was removed on 15th post-operative day following a retrograde cystography, which was negative for urinary leakages at the level of pouch-urethral anastomosis.

\section{Results}

The patient reported full day-time continence from about 2 weeks after the removal of the bladder catheter, using a single daily pad for self-assurance. Furthermore, the patient reported complete and fully satisfactory sexual intercourses, without any additional PDE-5 inhibitor or prostaglandin penile injections. No difficulties in maintaining the erection to completion sexual intercoursewas reported. Specific domains were analysed using International Index of Erectile Function 5 (IIEF-5), with the result of 24.8 years after the latter conversion, during latest clinical examination the patient was administered with 36-Item Short Form Health Survey SF-36 which revealed good-to-optimal results in each domain (Table 1).

Table 1: SF-36 scores reporting patient's results and average SF36 scores.

\begin{tabular}{|c|c|c|c|}
\hline & N. of Items & Total Score & Average Score \\
\hline Physical functioning & 10 & 900 & 90 \\
\hline Role functioning/physical & 4 & 300 & 75 \\
\hline $\begin{array}{c}\text { Role functioning/ } \\
\text { emotional }\end{array}$ & 3 & 300 & 100 \\
\hline Energy/fatigue & 4 & 240 & 60 \\
\hline Emotional well-being & 5 & 380 & 76 \\
\hline Social functioning & 2 & 150 & 75 \\
\hline Pain & 2 & 200 & 100 \\
\hline General health & 5 & 375 & 75 \\
\hline Health change & 1 & 75 & 75 \\
\hline
\end{tabular}

\section{Discussion}

Choice of UD after RC is based on different features including oncologic aspects, patients related co-morbidities such as age at surgery, renal and liver function, previous enteric surgery and patient preference. A proper patient selection is essential to achieve satisfactory QoL outcomes for any type of diversion. Patients with ON will need initial and sometimes long follow up, to increase time between clocked voiding and/or self-catheterization to gradually expand the bladder storage capacity, with seldom lasting nighttime incontinence [5,9]. On the other hand, patients with IC will experience a greater alteration of their self-body image, along with a long period required to comprehend an adequate stoma care. According to current literature, postoperative daytime and nocturnal continence after RC with ON ranges between 87-95\% and $72-95 \%$ respectively [10]. Moreover, postoperative erectile function $(\mathrm{EF})$ is another important factor which further impairs BC male individuals following RC. RC significantly affects EF recovery, as result of removal or damage of the neurovascular bundles.

When considering nerve-sparing RC, the two main outcomes are represented by the gain of a sufficient rate of EF recovery and a nonetheless adequate local cancer control [13]. In very selected patients with non-muscle invasive $\mathrm{BC}$, good pre-operative IIEF score and highly motivated to preserved sexual function, several 
sex-sparing techniques of RC have been proposed through the years, with potency rates between $75100 \%$ [12,13]. Several questionnaires have been proposed to measure QoL in patients who underwent RC evaluating physical, emotional, cognitive, work- and role- related and seldom spiritual aspects [7]. The SF36 is structured with 36 questions which considers eight aspects of HR-QoL and demographics with a 0-100 score. However, despite minimal differences in terms of QoL in general, patients with ON scored better in Role functioning and Role-emotional specific items than those with IC: the absence of a cutaneous stoma and therefore a preserved image of theirself, improved the state of mind in patients with a good life expectancy [13]. Furthermore, patients aged less than 65 with ON presented a better SF 36 score throughout the whole examination [14].

Our patient reported a fully recovered sexual potency right after the first surgical procedure, however, the presence of the cutaneous stoma impaired greatly his sexual, affective and personal sphere, with few sexual intercourses and impaired libido. Despite the lack of previous evidence of similar case described in literature, taking into account his motivations and according to his relatively young age, good performance and optimal oncological status, we chose to attempt the surgical conversion into a novel ON. No early nor late surgical complications were recorded at last follow up. Urinary continence was reached two weeks after bladder catheter removal, despite a 9 year-span of inhabited urethra. Furthermore, he reported a deep satisfaction concerning sexual activity and overall status. Despite the lack of clear evidences, a young and motivated BC patient with good life expectancy from an oncological and general standpoint could benefit from an "atypical" surgical conversion of IC into a ON, especially concerning the psychological sphere. We present an extremely singular case, with huge technical difficulties due to an altered abdominal anatomy following previous surgery. Beside oncological and medical outcomes in general, QoL has to be considered as an important endpoint when considering the most suitable type of diversion.

\section{References}

1. Stein JP, Lieskovsky G, Cote R, Groshen S, Feng AC, et al. (2001) Radical cystectomy in the treatment of invasive bladder cancer: long term results in 1054 patients. J Clin Oncol 19(3): 666-675.

2. Philip J, Manikandan R, Suresh Venugopal, John Desouza, Pradip M Javlé (2009) Orthotopicneobladder versus ileal conduit urinary diversion after cistectomy- a quality-of-life based comparison. Ann R Coll Surg Engl 91(7): 565-569.

3. Hautmann RE, Abol Enein H, Lee CT, Mansson W, Mills RD, et al. (2015) Urinary Diversion: How Experts Divert. Urology 85(1): 233-238.

4. Autorino R, Quarto G, Di Lorenzo G, De Sio M, Perdonà S (2009) Health related quality of life after radical cystectomy: Comparison of ileal conduit to continent orthotopicneobladder. Eur J Surg Oncol 35(8): 858864.

5. Schiavina R, Borghesi M, Guidi M, Vagnoni V, Zukerman Z, et al. (2013) Perioperative complications and mortality after Radical Cistectomy when using a standardized reporting methodology. Clin Genitourin Cancer 11(2): 189-197.

6. Lee RK, Abol Enein H, Artibani W, Bochner B, Dalbagni G, et al. (2014) Urinary diversion after radical cystectomy for bladder cancer: Options, patient selection, and outcomes. BJU Int 113(1): 11-23.

7. Clifford TG, Shah SH, Bazargani ST, Miranda G, Cai J, et al. (2016) Prospective evaluation of continence following radical cystectomy and othotopic urinary diversion using a validated questionnaire. J Urol 196(6): 1685-1691.

8. Studer UE, Zingg EJ (1997) Ilealorthotopic bladder substitutes. What we have learned from 12 years' experience with 200 patients. Urol Clin North Am 24(4): 781-793.

9. A Kretschmer, T Grimm, Buchner A, Grimm J, Grabbert M, et al. (2017) Prognostic features for objectively defined urinary continence after radical cystectomy and ileal orthotopic neobladder in a contemporary cohort. J Urol 197(1): 210-215.

10. Todenhofer T, Stenzl A, Schwentner C (2013) Optimal use and outcomes of orthotopic neobladder reconstruction in men and women. Current Opinion in Urology 23: 479-486.

11. Asgari MA, Safarinejad MR, Shakhssalim N, Soleimani M, Shahabi $A$, et al. (2013) Sexual function after non-nerve sparing radical cystoprostatectomy: a comparison between ileal conduit urinary diversion and orthotopicilealneobladder substitution. Int Braz J Urol 39(4): 474-483.

12. Muto G, Collura D, Rosso R, Giacobbe A, Muto GL, et al. (2014) Seminalsparing Cystectomy: Technical Evolution and Results Over a 20-Year Period. Urology 83(4): 856-861.

13. Ali AS, Hayes MC, Birch B, Dudderidge T, Somani BK, et al. (2015) Health related quality of life (HR- QoL) after cistectomy: comparison between orthotopicneobladder and ileal conduit diversion. Eur J Surg Oncol 41(3): 295-299.

14. Fujisawa M, Isotani S, Gotoh A, Okada H, Arakawa S, et al. (2000) Health related quality of life with orthotopicneobladder versus ileal conduit according to the SF-36 survey. Urology 55(6): 862-865.
ISSN: 2574-1241

DOI: 10.26717/BJSTR.2018.11.002101

Conti GM. Biomed J Sci \& Tech Res

This work is licensed under Creative

Commons Attribution 4.0 License

Submission Link: https://biomedres.us/submit-manuscript.php

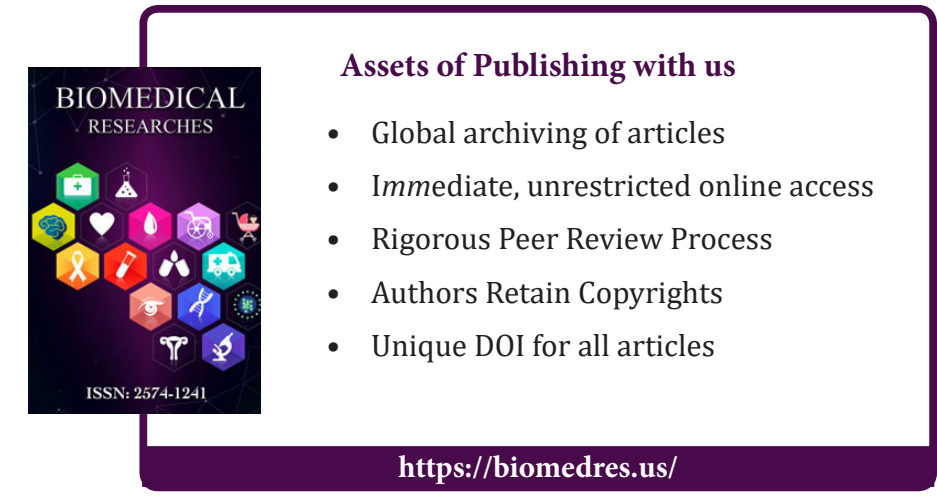

